\title{
A New Pluggable Framework for Centralized Routing in Wireless Sensor Network
}

\author{
Amir Mollanejad \\ Islamic Azad University- Jolfa Branch, Iran \\ amir.mollanejad@iauj.ac.ir \\ Amir Azimi Alasti Ahrabi \\ Islamic Azad University, Jolfa Branch, Iran \\ amir.azimi.alasti@gmail.com \\ Hadi Bahrbegi \\ Islamic Azad University, Jolfa Branch, Iran \\ Hadi.bahrbegi@gmail.com \\ Leyli Mohammad Khanli \\ Assistant professor Department of computer science University of Tabriz \\ Tabriz, IranL-khanli@tabrizu.ac.ir
}

\begin{abstract}
This paper presents a novel energy aware centralized dynamic clustering routing framework for large-scale Wireless Sensor Network (WSN). The main advantage of the proposed method is pluggability of clustering algorithms in the framework. It uses some clustering algorithms that some of their usages are new in this field. The clustering algorithms are K-means, FCM, UPC, GA, IGA and FGKA that run at base station used to identify cluster of sensors. Six clustering algorithms are evaluated in the framework and results of them are compared in three models named unicast, multicast and broadcast.
\end{abstract}

Index Terms-Wireless Sensor Network, Centralized Routing, Clustering Algorithm, Routing Framework.

\section{INTRODUCTION}

The main goal of wireless sensor network is collecting data and sending it to the Base Station (BS). One of the simplest and most preliminary ways for sending data to the BS is sending by direct way. Direct sending of data makes more loss of energy in sensors. In this way, nodes far away from the base station are destroyed earlier. To solve the problem, alternative algorithms were recommended that could reduce energy consumption in sensors. One of the solutions for this problem is clustering sensors and sending data to BS hierarchically. Clustering is an important mechanism in large multi-hop wireless sensor networks for obtaining scalability, reducing energy consumption and achieving better network performance [1]. Clustering a network to minimize the total distance is an NP-hard [2] problem [3].

In this paper a new pluggable framework for centralized routing in WSN is introduced and some clustering algorithms used to evaluate pluggability and performance of routing mechanism. The framework consists of three units that each of them has some duties. One of them is clustering unit that clustering algorithms are executed in this unit. The clustering algorithms are used to cluster sensors based on two parameter named position and residual energy of sensors. After constructing clusters by this unit, Cluster Heads (CHs) are identified and packets are sent across $\mathrm{CHs}$ to BS.

This paper is organized as follows: section 2, the related works of routing algorithms of WSN. Algorithms K-means, Fuzzy C-Means (FCM), Unsupervised Possibilistic Clustering (UPC), (Genetic Algorithm) GA, Improved Genetic Algorithm (IGA) and Fast Genetic Kmeans Algorithm (FGKA) will be discussed in section 3 . In section 4, proposed framework will be discussed. In section 5 the results of the new approach will be analyzed and finally, section 6 presents conclusion and future works.

\section{RELATED WORKS}

Heinzelman et al., proposed a clustering algorithm named LEACH(low-energy adaptive clustering hierarchy) [4]which is base of the most clustering protocols[5]. In WSN there are two ways on clustering, cluster head based clustering and location based [6]. In cluster head based protocols, the member nodes join in the cluster whose cluster head is the nearest. The cluster heads are important section of a cluster. LEACH protocol has some disadvantages. To solve these weaknesses DE_LEACH [7] is introduced by Li et al. The protocol uses differential evolution to optimize the cluster heads selection of LEACH protocol. 
SPIN [8] (Sensor Protocol for Information via Negotiation) is proposed to reduce amount of data messages while data sending to any node in the network. In this protocol meta-data information used to negotiate between nodes across 3-way handshaking. This avoids the transmission of redundant data in the network. A new method named DirQ is proposed in [9] that decreases energy consumption and quantity of sent messages. Kulkarni et al. proposed AIMRP protocol [10] that organizes the network into tiers around a base station, and progressively routes packets by forwarding them to a closer tier from the base station.

In the other work He et al. proposed new approaches that combines genetic algorithm and Fuzzy C-Means clustering algorithm to balance energy [11]. To divide cluster to equal size $\mathrm{Yu}$ et al. [12] presented a competition radius for cluster head selection.

Xiang et al. [13] proposed the clustering algorithm based on the best angle and the best single-hop distance, which can only be used in circle sensing area with base station in the center. In a corona-based WSN Ferng et al. proposed static clusters with dynamic structures by utilizing virtual points[14]. One of the limitations of this method is that the base station should be placed in center.

\section{CLUSTERING METHODS}

In this section, three groups of algorithms were presented to consider the application of protocol. These groups include: noise-resistant algorithms, heuristic algorithms, and evolutionary algorithms.

Heuristic algorithms use alternating optimization to cluster problem space. For example Fuzzy C-Means (FCM) [15] and K-Means [16] algorithms belongs to this category. The main problem of the algorithms is surrounded in local optimums but they have high execution speed.

Noise-resistant algorithms are ignored noisy points or outliers on the search space. In this paper possibilistic noise-resistant algorithms are used. This category of clustering algorithm is introduced by Krishnapuram et al. They proposed a new method named Possibilistic CMeans (PCM) algorithm [17] \& [18]. The main disadvantages of PCM are as follows: it generates coincident clusters and also it is very sensitive to initial points. UPC [19] is based on PCM algorithm and also resistant against noisy data. To solve problems of PCM, Yang and $\mathrm{Wu}$ proposed a new algorithm called UPC. It combines the objective function of FCM and two cluster validity indices to facilitate tuning of algorithm parameters.

An evolutionary algorithm (EA) uses mechanisms inspired by biological evolution, such as reproduction, mutation, recombination, and selection. Genetic algorithm is one of the EA that used in the clustering problem. In this paper three genetic algorithms based clustering method named GA, IGA, and FGKA are evaluated.

The main advantage of using of these algorithms is that they are not surrounded in local optimums. Generally they use some operators such as crossover, mutation and selection. IGA is introduced in [20] and uses an extra operator named K-Means. K-means algorithm is used to obtain initial population. This leads to improve the convergences of the algorithm. FGKA is similar to GA but it uses K-means algorithm after each mutation. Selection operator selects next population from output of the K-means algorithm. The evolution takes place until the termination condition is reached [21].

\section{PlugGable CENTRALIZED ROUTING FRAMEWORK}

In this section the new framework named PCRF is introduced. The framework consists of three units: Data Gathering and Energy Evaluation Unit (DGEEU), Clustering Unit (CU), and Notification Unit (NU). Fig. 1 shows the structure of the PCRF.

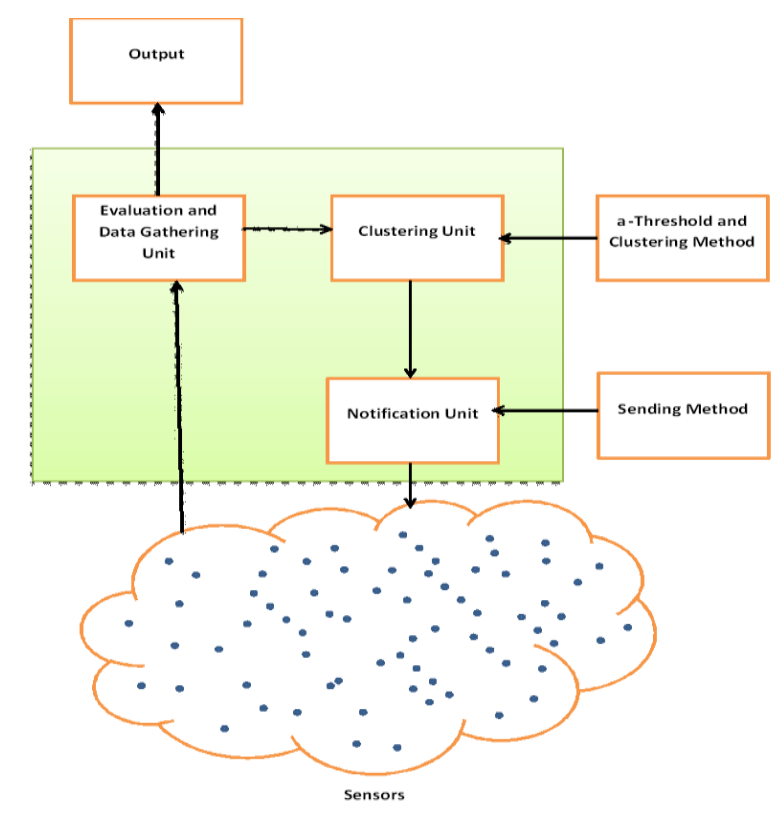

Fig 1. Structure of the PCRF

\section{A. Data Gathering and Energy Evaluation Unit}

This unit has two responsibilities. One of them is to gather required information about the sensors and the other is to decide whether collected information should be clustered or not. Required information is consists of sensed data, sensors position and residual sensors energy. Fig. 2 shows the algorithm of this unit. 
Input: Sensors information

Output: Sensed data, sensors position and residual energy

1. POSITIONS = new Array(),ENERGY = new Array();

2. for each sensor do:

a. if( sensors are mobile or first step)

i. Receive sensor position;

ii. $\quad$ put position in POSITIONS array;

b. Receive residual sensor energy and put it to ENERGY array;

c. Receive sensed data and send it to output;

3. OUTPUT_ENERGY = new Array();

4. if(sensors are mobile)

a. OUTPUT_POSITIONS = new Array ()$;$

5. for each energy in Energy array:

a. if(energy $>0)$

i. Add energy to OUTPUT_ENERGY;

ii. Add corresponding position in POSITIONS to OUTPUT_POSITIONS;

6. if(length of OUTPUT_ENERGY is not equal to zero)

a. Send OUTPUT_ENERGY array and OUTPUT_POSITIONS array to

Clustering Unit;

b. Run Clustering Unit;

c. Go to step 1;

else

d. finish.

Fig 2. Algorithm of Data Gathering and Energy Evaluation Unit

\section{B. Clustering Unit}

This unit is responsible to cluster all sensor nodes based on sensor positions and their residual energy. This unit is the core unit and pluggable section of the framework. Any clustering algorithm can be used in this unit. Note that each clustering algorithm should implement a specific interface. The interface should have a method that gets POSITIONS and ENERGY array from DGEEU unit then returns resulted clusters and their cluster heads. Cluster head is a nearest node to center of cluster (based on position and residual energy). When number of alive sensors are little than a threshold value, each alive sensor marked as Cluster Head and then forwarded to next unit. Fig. 3 shows the algorithm of this unit.

Each clustering method should find nearest sensor to the center of each cluster as Cluster Head sensor. Equation (1) shows this calculation.

$$
C H_{i}=\min \left(\operatorname{Dis} \tan c e\left(C_{i}, S\right)\right)
$$

Where $C_{i}$ is center point of cluster $I$ and $S$ is set of sensor vectors in cluster $\mathrm{i}$.

\section{Notification Unit}

This unit is responsible to notify all information about clusters and cluster heads to all sensors. This unit is simple unit that gets information about clusters and their heads from CU unit and sending method from outside of framework. In this paper three sending methods are used named Broadcast Method, Multicast Method and Unicast Method.

Input: POSITIONS, ENERGY arrays, $\alpha$ threshold and Clustering Algorithm

Output: CLUSTERS and CLUSTER HEADS

1. CLUSTER_RESULT = new array;

2. if(length $(E N E R G Y)>\alpha)$

a. Call corresponding Clustering method of input Clustering Algorithm with parameters POSITIONS and ENERGY arrays;

b. Do clustering operation;

c. Get CLUSTERS and CLUSTER_HEADS arrays from above method;

d. for each Cluster Head in CLUSTERS_HEADS array do

$i$. $\quad$ temp $=$ find corresponding cluster nodes (index of them in array);

ii. for each element in temp do:

1. element value $=$ index of corresponding Cluster Head; else

e. for $i=0$ to $i<$ length $(E N E R G Y) d o$

$i$. Add $i$ to

CLUSTERS_RESULT array;

3. Send CLUSTERS_RESULT to Notification Unit.

Fig 3. Algorithm of Clustering Unit

\section{1) Broadcast Method}

This method makes an array for showing the structures of cluster that includes corresponding head-clusters of each cluster. The index of this array indicates the sensor ID. By receiving controlling packet, each sensor reads array value corresponds to its ID from array. By reading the value, sensor finds Cluster Head ID if this ID is equal to own ID that sensor is selected as $\mathrm{CH}$. Each sensor must send collected data to head-cluster. Fig. 4 shows an example of this controlling packet.

\begin{tabular}{|l|l|l|l|l|l|l|l|l|l|}
\hline Index/ID & 1 & 2 & 3 & 4 & 5 & 6 & 7 & 8 & 9 \\
\hline $\mathrm{CH}$ & 3 & 7 & 3 & 3 & 7 & 3 & 7 & 7 & 7 \\
\hline
\end{tabular}

Fig 4. Broad cast packet

The above figure indicates information of two clusters. Sensors with IDs 1, 3, 4 and 6 belong to first cluster with $\mathrm{CH}$ ID 3 and sensors with IDs 2, 5, 7, 8 and 9 belong to second cluster with $\mathrm{CH}$ ID7. As you can see, these clusters are separated based on the number of the headcluster. The base station chose the sensors 3 and 7 as the head-cluster for two clusters. The following equation is used to determine the length of controlling packet. 


$$
\text { PacketLength }_{\text {Broadcast }}=n \times l
$$

Where $\mathrm{n}$ is the number of sensors and 1 is the size of each entry of array in byte.

\section{2) Multicast Method}

In this method, according to clusters some packets containing array are constructed and directly sent by base station to correspond cluster. This message is an array of sensors of each cluster and its corresponding head-cluster. Fig. 5 shows a sample packet for a cluster, Last element in the array is $\mathrm{CH}$ ID. The first five elements of array indicate cluster elements. The sixth element of array is $\mathrm{CH}$. All sensors in the cluster receive this packet and find own ID from array then read last element and find who the $\mathrm{CH}$ is. It is possible some sensors receive sent packet of neighbor clusters. Also, by considering array elements, they find out that belong to this cluster or not. The length of array is related to number of sensors in each cluster.

\begin{tabular}{|l|l|l|l|l|l|}
\hline 5 & 4 & 62 & 2 & 11 & 4 \\
\hline
\end{tabular}

Fig 5. Multicast packet

\section{3) Unicast Method}

In this method small controlling packets contains $\mathrm{CH}$ ID of each element are directly sent by base station to each sensor. If received ID in the packet is equal to own ID of sensor therefore the sensor notice that is selected as $\mathrm{CH}$. After this phase each element of cluster sends a message to $\mathrm{CH}$ to construct TDMA [1] controlling packet by $\mathrm{CH}$. TDMA algorithm is also used in broadcast and multicast protocols.

\section{NETWORK MODEL AND ASSUMPTIONS}

In this section some assumptions and data communication algorithms are investigated. We make following assumptions for our sensor network:

1. Network environment is in a two Dimensional space with a specific width and length and sensors are placed in positions with a specific width and length,

2. Nodes are dispersed randomly following a Uniform Distribution and they equipped with a GPS set,

3 . The energy of sensor nodes cannot be recharged,

4. The nodes are capable of transmitting at variable power levels depending on the distance to the receiver as in $[2,3]$. For instance, the MICA Motes use MSP430 [3, 4] series micro controller which can be programmed to 31 different power levels,

5. BS is distinguished from other nodes by its unlimited energy supply,

6. BS is capable to send packet in three model; unicast, multicast, broadcast,

7. The connectivity requirement achieved being used by an adaptive transmits power, where node increases its transmission power to reach $\mathrm{CH}$.

Radio model in [5] is selected. In this model, for a short transmission range such as within clusters, the energy consumed by a transmit amplifier that is proportional to $\mathrm{d}^{2}$, where $\mathrm{d}$ is the distance between nodes. However, for a long transmission range such as from a cluster head to the base station, the energy consumed that is proportional to $\mathrm{d}^{4}$. By using given radio model, the energy consumed $\mathrm{ET}_{\mathrm{ij}}$ to transmit a message of 1-bits length from a node $i$ to a node $j$ is given by (1) and (2) for long and short distances, respectively [4].

$$
\begin{aligned}
E_{T x}(l, d) & =E_{T x-\text { elec }}(l)+E_{T x-a m p}(l, d) \\
& = \begin{cases}l E_{\text {elec }}+l \epsilon_{\mathrm{fs}} d^{2}, & d<d_{o} \\
l E_{\text {elec }}+l \epsilon_{\mathrm{mp}} d^{4}, & d \geq d_{Q} .\end{cases}
\end{aligned}
$$

Where $E_{\mathrm{Tx}}$ is total transmit energy and $E_{\text {elec }}$ is the electronic energy. $\varepsilon_{\mathrm{mp}}$ is the energy required by transmit amplifier to maintain an accepable signal to noise ratio in order to transfer data message reliably. $\varepsilon_{\mathrm{fs}}$ is transmit parameter related to the free-space [2002 helsman]. To receive the message, the radio is:

$$
E_{R X}=E_{\text {elec }} \times l
$$

Where $E_{R X}$ is energy cost incurred in the receiver of the destination node [4].

\section{EXPERIMENTAL RESULTS}

In this section results of the framework with six clustering algorithms and three sending methods are evaluated and compared to LEACH protocol. MATLAB software is used to simulate the framework and other protocols.

Performance is evaluated by quantitative metrics of network lifetime, average energy dissipation, total data messages successfully delivered, First Node Dies (FND), Half of the Nodes alive (HNA) [6] and number of nodes that are alive. We consider $\mathrm{N}$ nodes that randomly dispersed in $200 \times 200$ of square fields with a single BS are located at $(50,175)$. Table 1 shows the simulations parameters. Each simulation result shown below is the average of 20 independent experiments where each experiment uses a different randomly-generated uniform topology of sensor nodes. Note that in all experimental results sending methods have no effect on LEACH protocol.

Table 1. PARAMETERS USED IN SIMULATIONS

\begin{tabular}{cc}
\hline Value & Parameter \\
\hline$(0,0) \sim(200,200)$ & Network Filed \\
$100 \sim 500$ & Node numbers \\
$40 \mathrm{~m}$ & Cluster radius $r$ \\
$15 \mathrm{~m}$ & Sensing radius $r \mathrm{~s}$ \\
$(50,175)$ & Sink position \\
$0.5 \mathrm{~J}$ & Initial energy \\
2000 Bytes & Data packet size \\
10 Bytes & Broadcast packet size \\
$0.01 \mathrm{~J}$ & Ethreshold
\end{tabular}




\section{A) Alive Sensors in Each Round}

This measure investigates number of alive sensors in each round in three methods of sending data.

\section{1) Broadcast Method}

Fig. 1 shows network life time and number of alive nodes across broadcasting method of framework with six clustering algorithms and compares them to LEACH protocol. The shortest network life time belongs to LEACH protocol with 1455 rounds and the longest one belongs to FGKA with 2675 rounds. As shown in Fig. 1, in LEACH protocol after passing a period of time, sensors start to die with more speed and network energy disappear with steep slope, but in other algorithms this slope is gentle and it can be said that the network is more stable.

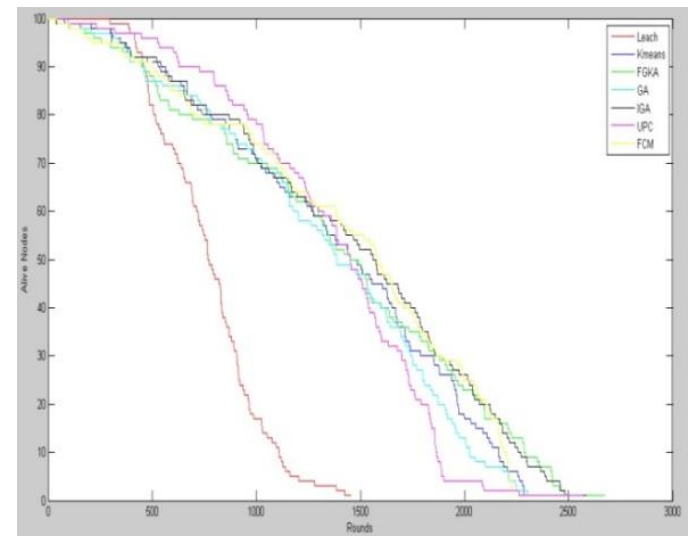

Fig 6. Number of live node per round in broadcast model

\section{2) Multicast Method}

Fig. 2 shows network life time and number of live nodes across multicasting method of framework with six clustering algorithms. The result of the framework compared with LEACH protocol. Same as the broadcasting method, the shortest network life time belongs to LEACH protocol with 1426 rounds and the longest one belongs to UPC with 2844 rounds. As shown in Fig. 2, same as broadcasting method, in LEACH protocol, sensors start to die with more speed and network energy disappear with steep slope, but in other algorithms this slope is gentle and it can be said that the network is more stable.

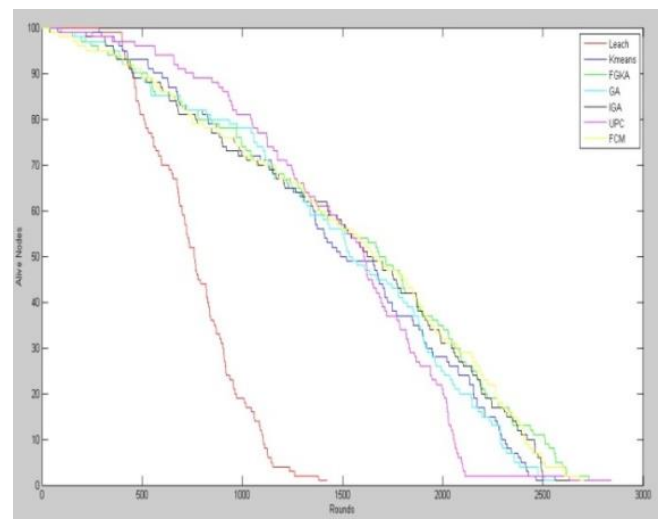

Fig 7. Number of alive node per round in multicast model

\section{3) Unicast Method}

Fig. 3 shows network life time and alive nodes across unicasting method of framework. The results also compared with LEACH protocol result. Same as the broadcasting and multicasting methods, the shortest network life time belongs to LEACH protocol with 1455 rounds and the longest one belongs to FCM with 3161 rounds. As shown in Fig. 3, same as other methods, in LEACH protocol, sensors start to die and network energy suddenly disappeared. The results show that in other algorithms, network is more stable than LEACH protocol.

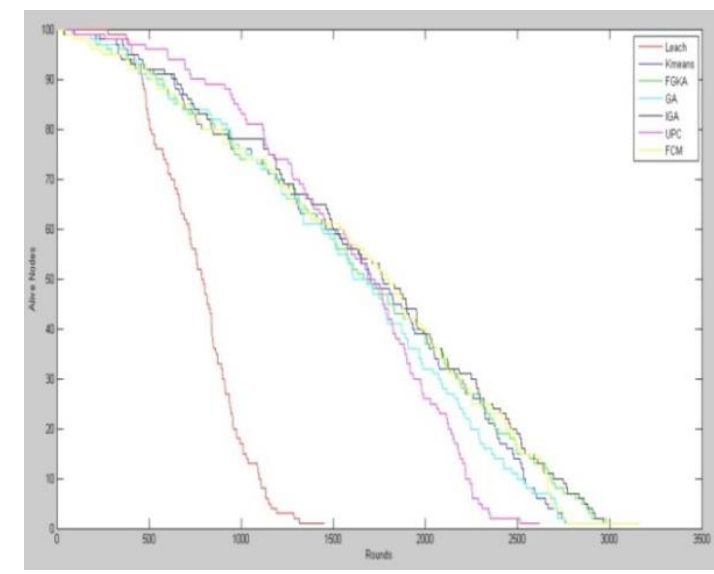

Fig 8. Number of alive node per round in unicast model

Initial energy of each sensor is 0.5 joule. In this section, we compare the results of simulation for average energy dissipation for proposed framework and LEACH protocol.

\section{B) Average energy dissipation}

\section{1) Broadcast Method}

Fig. 4 shows average energy dissipation in each round in broadcast method. As shown in Fig. 4, in LEACH protocol all energy of sensors is consumed in 1455 rounds but in FGKA that is 2675 rounds.

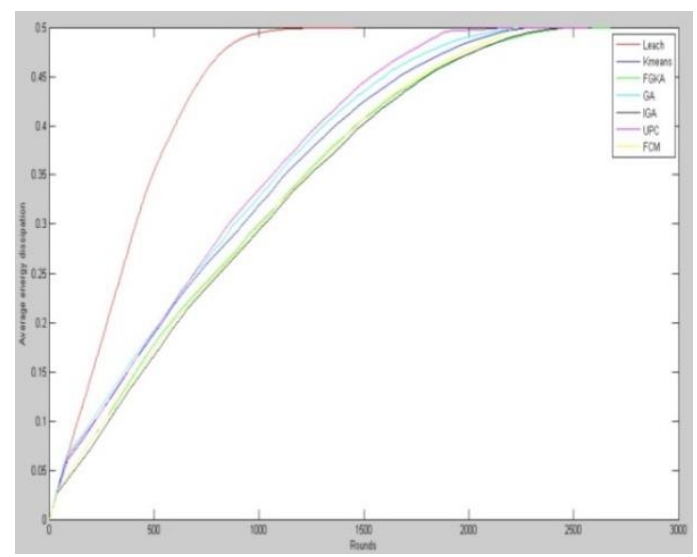

Fig 9. Average energy dissipation per Round in broadcast model

\section{2) Multicast Method}

Fig. 5 shows average energy dissipation in each round in multicasting method. In LEACH protocol and UPC all energy of sensors are consumed in 1455 and 2844 rounds respectively. 


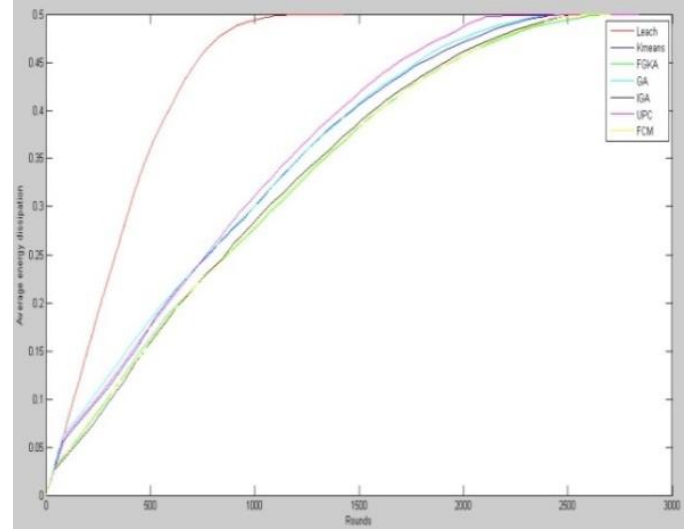

Fig 10. Average energy dissipation per Round in multicast model

\section{3) Unicast Method}

Fig. 6 shows average energy dissipation in each round in unicasting method. In LEACH protocol and FCM all energy of sensors are consumed in 1455 and 3161 rounds respectively.

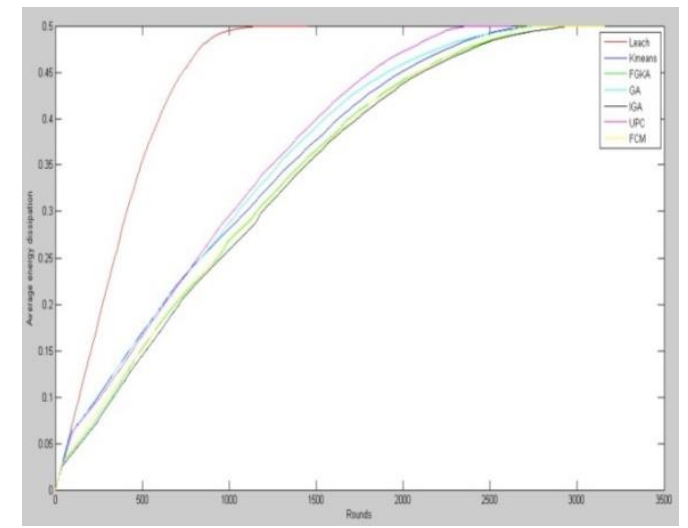

Fig 11. Average energy dissipation per Round in unicast model

\section{C) Number of data messages received at the base} station

In this section all received data packets in base station in each round are compared with together in different sending methods.

\section{1) Broadcast Method}

Fig. 7 shows received data packets in base station for broadcasting method. As shown in the figure, all received data packets in base station in LEACH protocol are 77586 packets. For this measure highest value belongs to IGA with 145997 packets.

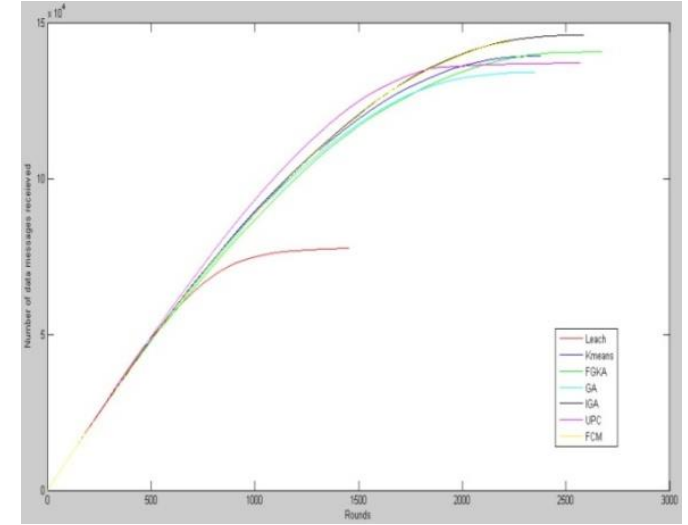

Fig 12. Comparison total number of data messages received at the base station in broadcast model between LEACH and proposed algorithms

\section{2) Multicast Method}

Fig. 8 shows received data packets in base station for multicasting method. This measure is 76694 and 156184 in LEACH protocol and FGKA respectively.

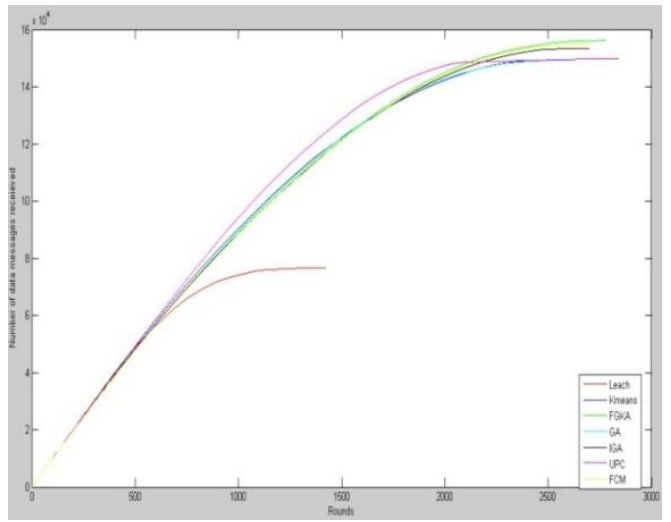

Fig 13. Comparison total number of data messages received at the base station in multicast model between LEACH and proposed algorithms

\section{3) Unicast Method}

Fig. 9 shows received data packets in base station for unicasting method. This measure is 77289 and 170717 in LEACH protocol and IGA respectively.

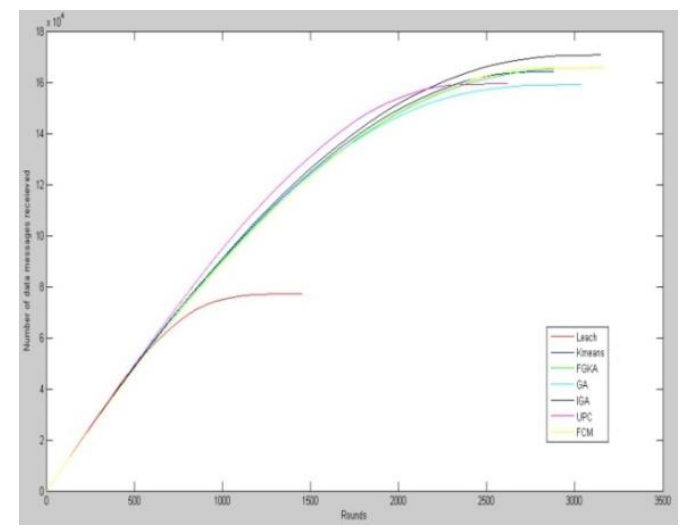

Fig 14. Comparison total number of data messages received at the base station in unicast model between LEACH and proposed algorithms 


\section{D) The First Node Die and Half of Nodes Alive}

This section compares two criteria of the First Node Die (FND) and Half of Nodes Alive (HNA) by proposed framework and LEACH protocol [7]. FND indicates round of the first dead node. HNA also, indicates round of half of live nodes.

\section{1) Broadcast Method}

Fig. 10 shows comparison of results for FND metric. As shown in the figure, the first node in LEACH protocol dies in round 295 and in UPC method is round 95.

Fig. 11 shows the comparison results for HNA of LEACH protocol and proposed framework algorithms for methods in broadcasting method. As shown in the figure, LEACH protocols and FCM have values 767 and 1579 respectively. It means that FCM result is nearly two times of the results in LEACH protocol. This results show in LEACH protocol, sensors are dead with high speed. Also the stability of the framework is approved by these metric values.

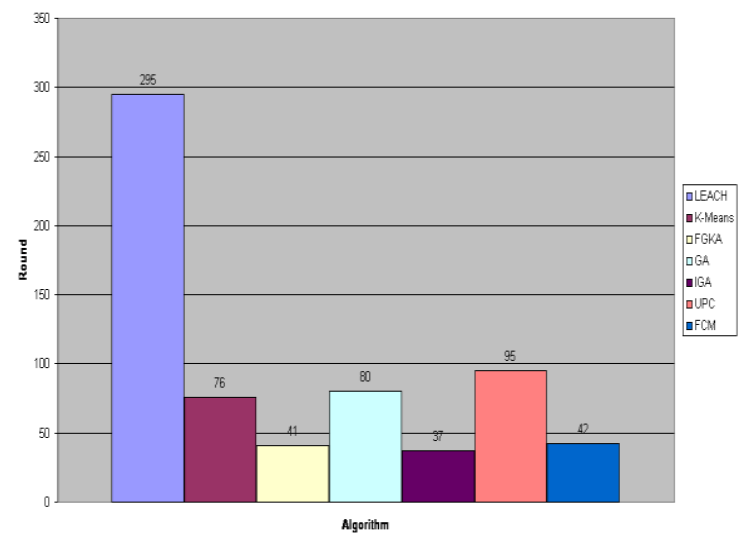

Fig 15. Network life time comparison using FND criteria in broadcast model between LEACH and proposed algorithms

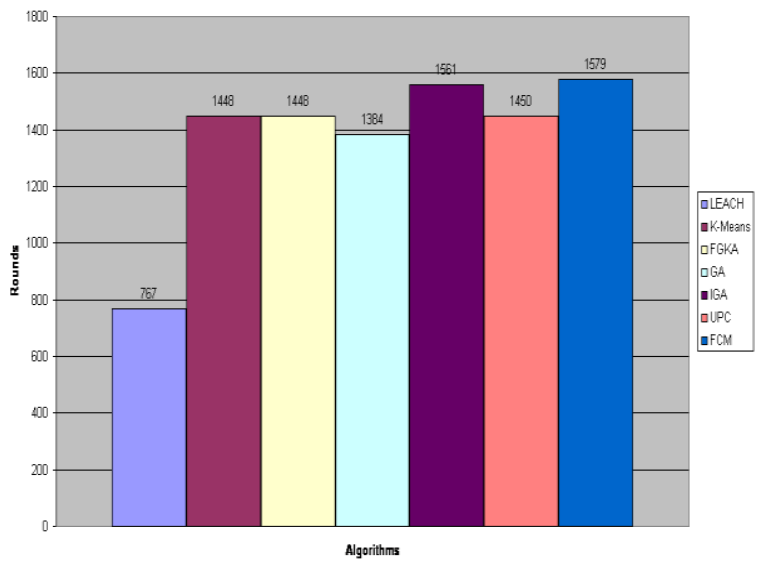

Fig 16. Network life time comparison using HNA criteria in broadcast model between LEACH and proposed algorithm

\section{2) Multicast Method}

Fig. 12 shows comparison of results for FND metric. As shown in the figure, the first node in LEACH protocol dies in round 284 and in UPC method is round 95.

Fig. 13 shows the comparison results for HNA of LEACH protocol and proposed framework algorithms for methods in multicasting method. As shown in the figure, LEACH protocols and FGKA have values 759 and 1681. Same as the broadcasting method, in LEACH protocol, sensors are dead with high speed.

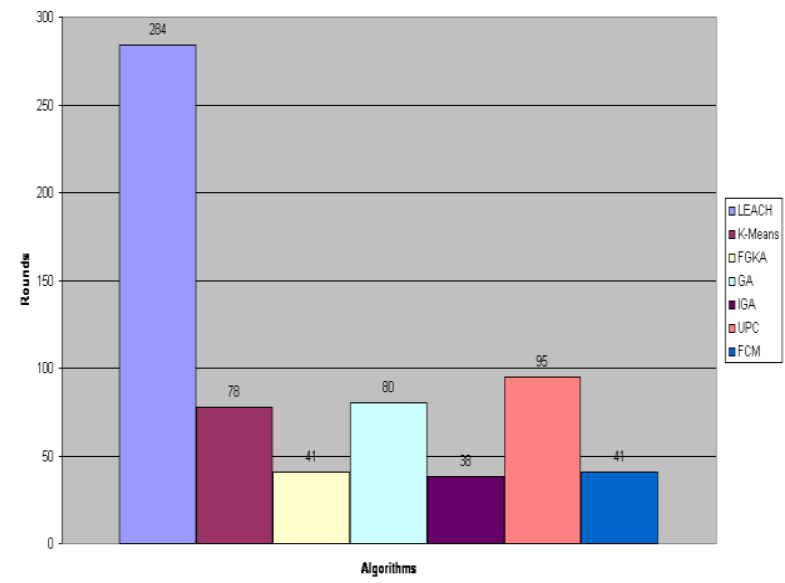

Fig 17. Network life time comparison using FND criteria in multicast model between LEACH and proposed algorithms

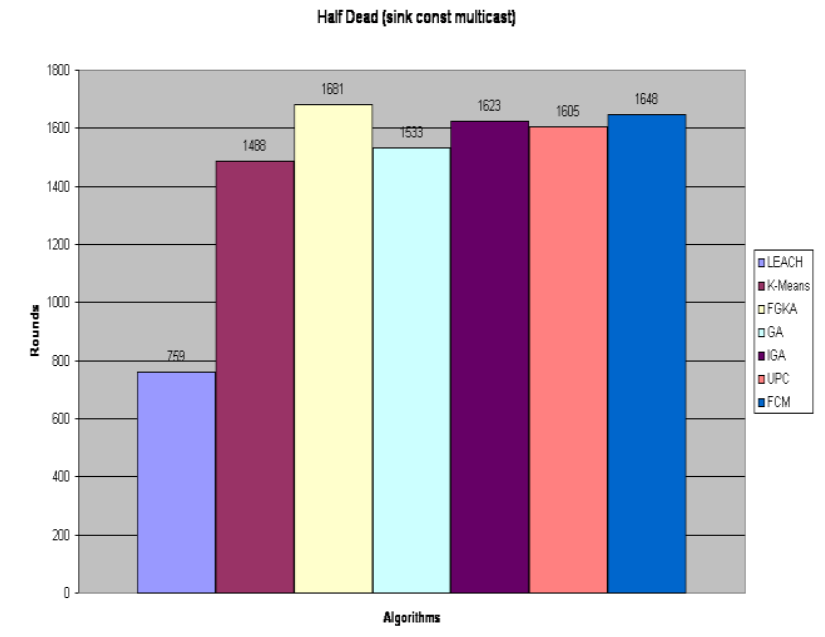

Fig 18. Network life time comparison using HNA criteria in multicast model between LEACH and proposed algorithm

\section{3) Unicast Method}

Fig. 14 shows comparison of results for FND metric. As shown in the figure, the first node in UPC algorithm dies in round 97 and in LEACH protocol is round 276.

Fig. 15 shows the results for HNA of LEACH protocol and proposed framework algorithms for unicasting method. As shown in the figure, LEACH protocols and FCM have values 767 and 1792. Same as other methods, in LEACH protocol, sensors are dead with high speed. 


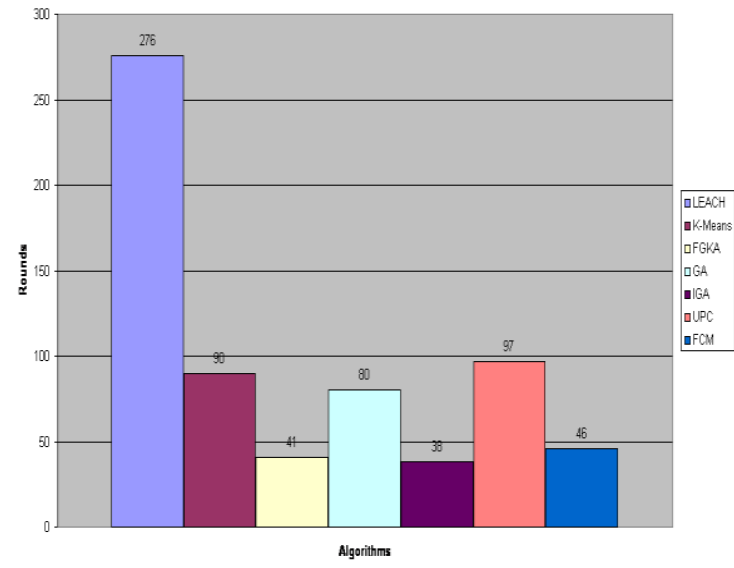

Fig 19. Network life time comparison using FND criteria in unicast model between LEACH and proposed algorithms

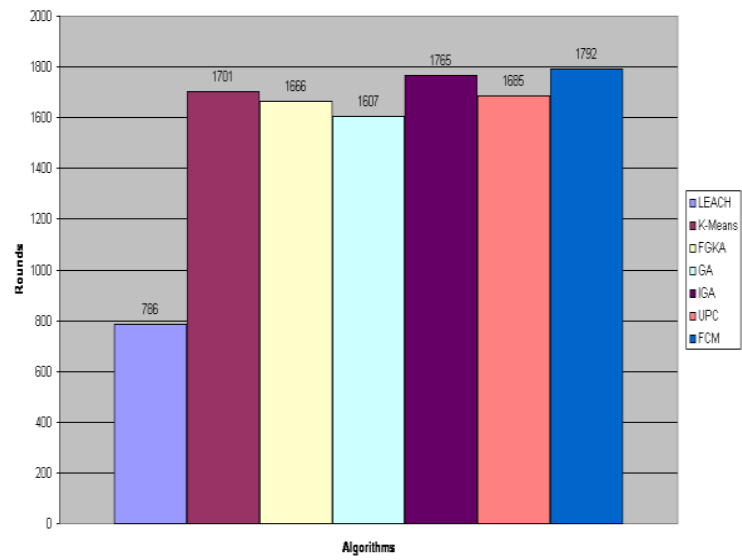

Fig 20. Network life time comparison using HNA criteria in unicast model between LEACH and proposed algorithm

\section{CONCLUSION AND FUTURE WORK}

In this paper a new pluggable framework for centralized routing in WSN is proposed. To test performance of the system and showing pluggability feature of the system six clustering algorithms are implemented. In the experimental results, the proposed framework is evaluated by three packet sending methods from base station to the sensors. Results of the six clustering algorithms are compared with each other and LEACH protocol. Results show that network life time and network stability is improved by using proposed framework.

Using minimum spanning tree to sending collected data from $\mathrm{CHs}$ to BS could be one of future works of this paper. The tree constructed by BS based on positions and residual energies of $\mathrm{CHs}$ and then send tree information to all CHs.

\section{REFERENCES}

[1] J. Hamalainen, et al., "System for transmitting packet data in digital cellular time division multiple access (TDMA) air interface," ed: Google Patents, 1997.
[2] S. Hussain, et al., "Genetic algorithm for energy efficient clusters in wireless sensor networks," in Information Technology, 2007. ITNG'07. Fourth International Conference on, 2007, pp. 147-154.

[3] T. Instruments, "MSP430x13x, MSP430x14x, MSP430x14x1 MIXED SIGNAL MICROCONTROLLER," Post Office Box, vol. 655303, 2004.

[4] W. B. Heinzelman, et al., "An application-specific protocol architecture for wireless microsensor networks," Wireless Communications, IEEE Transactions on, vol. 1, pp. 660-670, 2002.

[5] W. R. Heinzelman, et al., "Energy-efficient communication protocol for wireless microsensor networks," in System Sciences, 2000. Proceedings of the 33rd Annual Hawaii International Conference on, 2000, p. 10 pp. vol. 2.

[6] B. Thomas and F. Hoffmeister, "Global optimization by mans of evolutionary alghorithms," Random Search as Method for Adaptation and Optimization of Complex Systems, edited by: AN Anamoshkin, Kras-Nojarsk Space Technology University, pp. 17-21, 1996.

[7] A. Mollanejad, et al., "DBSR: Dynamic base station Repositioning using Genetic algorithm in wireless sensor network," in Computer Engineering and Applications (ICCEA), 2010 Second International Conference on, 2010, pp. 521-525.

[8] T. Anker, et al., "Efficient clustering for improving network performance in wireless sensor networks," in Wireless Sensor Networks, ed: Springer, 2008, pp. 221236.

[9] M. Chatterjee, et al., "WCA: A weighted clustering algorithm for mobile ad hoc networks," Cluster Computing, vol. 5, pp. 193-204, 2002.

[10] X. Lin and I. Stojmenovic, "Power-aware routing in ad hoc wireless networks," SITE, University of Ottawa, TR98, vol. 11, 1998

[11] W. R. Heinzelman, et al., "Energy-efficient communication protocol for wireless microsensor networks," in System Sciences, 2000. Proceedings of the 33rd Annual Hawaii International Conference on, 2000, p. 10 pp. vol. 2.

[12] K. Akkaya and M. Younis, "A survey on routing protocols for wireless sensor networks," Ad hoc networks, vol. 3, pp. 325-349, 2005.

[13] J. Chen, et al., "A Centralized Balance Clustering Routing Protocol for Wireless Sensor Network," Wireless Personal Communications, pp. 1-12, 2013.

[14] X. Li, et al., "A differential evolution-based routing algorithm for environmental monitoring wireless sensor networks," Sensors, vol. 10, pp. 5425-5442, 2010.

[15] W. R. Heinzelman, et al., "Adaptive protocols for information dissemination in wireless sensor networks," in Proceedings of the 5th annual ACM/IEEE international conference on Mobile computing and networking, 1999, pp. 174-185.

[16] S. Chatterjea, et al., "DirQ: A Directed Query Dissemination Scheme for Wireless Sensor Networks," in Wireless and Optical Communications, 2006.

[17] S. Kulkarni, et al., "An address-light, integrated MAC and routing protocol for wireless sensor networks," 2005.

[18] S. He, et al., "A Clustering Routing Protocol for Energy Balance of WSN based on Genetic Clustering Algorithm," IERI Procedia, vol. 2, pp. 788-793, 2012.

[19] J. Yu, et al., "A cluster-based routing protocol for wireless sensor networks with nonuniform node distribution," 
AEU-International Journal of Electronics and Communications, vol. 66, pp. 54-61, 2012.

[20] X. Min, et al., "Energy efficient clustering algorithm for maximizing lifetime of wireless sensor networks," AEUInternational Journal of Electronics and Communications, vol. 64, pp. 289-298, 2010.

[21] H.-W. Ferng, et al., "Energy-efficient routing protocol for wireless sensor networks with static clustering and dynamic structure," Wireless Personal Communications, vol. 65, pp. 347-367, 2012.

[22] J. C. Bezdek, et al., "FCM: The fuzzy c-means clustering algorithm," Computers \& Geosciences, vol. 10, pp. 191203, 1984.

[23] K. Krishna and M. Narasimha Murty, "Genetic K-means algorithm," Systems, Man, and Cybernetics, Part B: Cybernetics, IEEE Transactions on, vol. 29, pp. 433-439, 1999.

[24] R. Krishnapuram and J. M. Keller, "A possibilistic approach to clustering," Fuzzy Systems, IEEE Transactions on, vol. 1, pp. 98-110, 1993.

[25] R. Krishnapuram and J. M. Keller, "The possibilistic cmeans algorithm: insights and recommendations," Fuzzy Systems, IEEE Transactions on, vol. 4, pp. 385-393, 1996.

[26] M.-S. Yang and K.-L. Wu, "Unsupervised possibilistic clustering," Pattern Recognition, vol. 39, pp. 5-21, 2006.

[27] L. Fuyan, et al., "An improved genetic approach," in Neural Networks and Brain, 2005. ICNN\&B'05. International Conference on, 2005, pp. 641-644.

[28] Y. Lu, et al., "FGKA: A fast genetic k-means clustering algorithm," in Proceedings of the 2004 ACM symposium on Applied computing, 2004, pp. 622-623.

[29] J. Hamalainen, et al., "System for transmitting packet data in digital cellular time division multiple access (TDMA) air interface," ed: Google Patents, 1997.

[30] S. Hussain, et al., "Genetic algorithm for energy efficient clusters in wireless sensor networks," in Information Technology, 2007. ITNG'07. Fourth International Conference on, 2007, pp. 147-154.

[31] T. Instruments, "MSP430x13x, MSP430x14x, MSP430x14x1 MIXED MICROCONTROLLER," Post Office Box, vol. 655303, 2004.

[32] W. B. Heinzelman, et al., "An application-specific protocol architecture for wireless microsensor networks," Wireless Communications, IEEE Transactions on, vol. 1, pp. 660-670, 2002.
[33] B. Thomas and F. Hoffmeister, "Global optimization by mans of evolutionary alghorithms," Random Search as Method for Adaptation and Optimization of Complex Systems, edited by: AN Anamoshkin, Kras-Nojarsk Space Technology University, pp. 17-21, 1996.

[34] A. Mollanejad, et al., "DBSR: Dynamic base station Repositioning using Genetic algorithm in wireless sensor network," in Computer Engineering and Applications (ICCEA), 2010 Second International Conference on, 2010, pp. 521-525.

Amir Mollanejad, M.S. in Software Engineering, Islamic Azad University, Shabestar Branch, 2010 has experience on Wireless Sensor Network (WSN) and Artificial Intelligence. He is currently headquarters of Computer Science Studies in Islamic Azad University of Jolfa International Branch.

Amir Azimi Alasti Ahrabi. He was born in 1983. He received the B.S. and M.S. degrees in software engineering from University of Payamenour and Islamic Azad University, in 2007 and 2010 respectively. His research areas are information security, and artificial intelligence.

Hadi Bahrbegi, M.S. in Software Engineering, Islamic Azad University, Shabestar Branch, 2010 has experience in Artificial Intelligence and Computer Security; especially in Intrusion Detection Systems (IDS) field and published more than 12 journal and conference articles. $\mathrm{He}$ is currently studies Distributed Artificial Intelligence.

Leili Mohammad Khanli, received her B.S. (1995) from Shahid Beheshti University Tehran, Iran,M.S. (2000) from IUST (Iran University of Science and Technology) University and a Ph.D. degree (2007) from IUST (Iran University of Science and Technology) University. All are in computer engineering. She is currently associate professor in the Department of Electrical and Computer Engineering at University of Tabriz. Her research interests include cloud computing and Quality of Service management.

How to cite this paper: Amir Mollanejad, Amir Azimi Alasti Ahrabi, Hadi Bahrbegi, Leyli Mohammad Khanli,"A New Pluggable Framework for Centralized Routing in Wireless Sensor Network", IJCNIS, vol.6, no.12, pp.28-36, 2014. DOI: 10.5815/ijcnis.2014.12.04 